\title{
Analysis Role of Abstract Thinking in Pipa Performance
}

\author{
Cong Zheng
}

The school of Music, Luoyang Normal University, Luoyang, 471934, China

\author{
Keywords: Abstract thinking; Pipa playing; Feeling; Control
}

\begin{abstract}
Abstract thinking is a complex and advanced form of human thinking. In playing lute, for it through the rational grasp of acoustics, mechanics, physiology and other disciplines of natural law to guide the training of playing techniques, the cognition of Pipa music creation background, musical structure analysis of music, and the appropriate control on the performance, has guide an important role. It introduces pipa playing to the track of science, systematization and standardization.
\end{abstract}

\section{Introduction}

The pipa occupies a very important position in the traditional Chinese plucked musical instruments. After more than 1000 years of spread and development, the pipa has become more and more perfect in form and repertoire, rich in techniques, distinctive personality, unique charm and varied timbre [1]. At the same time, the artistic expression of its unique charm increases the appeal of music, enriches the band's color, and makes it an indispensable plucking instrument in the band.

The timbre of the pipa is composed of natural timbre and changing timbre. The natural timbre is constructed from the material of the instrument itself. The natural timbre also requires the performer to use a scientific and correct playing method to play a relaxed, thick, round, bright and transparent subject tone, which is neutral, pure, and original [2]. No modification of the basic sound characteristics. The change of timbre refers to the players on the basis of the basic state of pronunciation, the use of a variety of playing techniques to change the main tone produced.

Abstract thinking is an advanced and complex form of thinking of human beings. For music learners, only when he has certain musical knowledge and experience, have rich music practice, music perceptual image and abstract image feeling, In order to gradually master and use music abstract thinking.

First of all, we must strengthen students' appreciation of music works and their mastery of music works. Each piece of music has a certain social background and the life experience of music composers. The characteristics of character are directly related to artistic experience. Generally speaking, the feelings and experiences of composers are directly influenced by their integration into the musical works, thus directly affecting the connotation of the works. Therefore, in the teaching process of pipa, It is necessary to strengthen students' mastery of musical works.

Abstract thinking in pipa performance is a form of thinking that guides pipa performance by means of rational methods such as concept, judgment, deduction and induction. It is general and indirect. It requires the performer to handle correctly the relationship between emotion and reason, and to represent the music rationally on the basis of understanding and experience.

\section{The Role of Abstract Thinking in the Process of Pipa Playing and Learning}

The art of pipa performance is the organic coordination of various combinations of techniques derived from the two systems of the right and the left in the performance techniques; by reasonable, measured, purposeful, on the basis of the basic state of pronunciation, To change the tone, tone quality, amplitude, intensity, speed, etc., to produce rich sound, to express emotions, describe artistic conception, depict images, and realize various requirements for artistic expression. In the art of pipa playing, the change of timbre is an important means to express the art of pipa playing, and the change of timbre is based on the need of the expression of music emotion.

As a rational form of thinking, abstract thinking plays an important guiding role in the process of pipa playing and learning: 
The laws of acoustics, mechanics, physiology and other natural subjects play an important guiding role in the training of pipa playing techniques, and these laws need to be analyzed and understood by abstract thinking.

Pipa playing is a complex musical activity; it needs to mobilize the various functions of the body, through the rational performance of the movement, the music content needs to produce a variety of acoustics [3]. We must make use of abstract thinking to analyze, understand and master the laws of acoustics, mechanics, physiology and other natural disciplines needed for performance. First, we must understand the physiological structure of the human body, understand the principle of mechanical production, give reasonable play to the functions of various parts of the human body, and freely make technical actions; second, we should understand the physical structure of the human body and the physical conditions of the pipa. We also have the knowledge of acoustics so that the sound emitted by the vibration of the string is as good as possible. In training, we have to study which parts of each action take part in the movement (finger, wrist, forearm, arm, etc.).

What is the form of these movements (flexion and extension, rotation, etc.) so that you can reasonably use your strength (rather than exertion) in training, that is to say, the part of the movement is active, the part that should not be moving, for example, the wheel finger, The main movements of the fingers are extension and flexion (with a minimal degree of rotation). The extension requires that the fingernails contract with greater force at the moment they pass through the strings, and that the flexor relax. If the muscles involved in the flexion of the finger also contract in order to form a confrontation, the extension will be difficult. Not enough relaxation of the finger will make the finger clumsy, stiff and inelastic. For example, "the weight of the arm" is also an extremely important concept in performance, and must be clearly understood.

If the weight of the arm and other parts cannot be used correctly and reasonably in application, and if you do not understand that, in any case, when the finger acts on the string, it will always contain the principle of the weight change of the arm and the like, and you will not be able to correctly grasp the volume and timbre [4]. The understanding and mastering of this physiological and mechanical knowledge can not be separated from rational abstract thinking.

With the help of abstract thinking, we can know the life of the composer, artistic accomplishment, the motives and purposes of Pipa music creation, the background of the works and the public's evaluation, so that the performers can better grasp the emotional latitude of the music and lay a solid foundation for their rational performance.

Pipa is a kind of national musical instrument with complicated playing techniques and profound cultural background. After 2,000 years of spread and evolution, pipa music has gradually formed a unique and systematic playing technique and theory. Therefore, when learning and playing the pipa, the performer first uses the relatively stable theoretical knowledge system of the pipa, which has been formed after countless explorations and studies by the predecessors. In order to recognize and remember the pipa works, the structure and function of the works can be expressed in the mind, which mainly depends on abstract thinking.

The performer must first of all recognize the composer's life, outlook on life, outlook on art, direct writing motivation and purpose of the work, the era reflected in the work, the cultural background, and the public's recognition of the criticism, debate, and so on [5] during the premiere [6]. Only by truly understanding the composer's obvious intention, incarnating himself as the second creator of the work and respecting the historical individuality of the work, can he lay a solid foundation for the final truthfulness of the performance.

For example, the solo "overlord and disarm armour" is a famous traditional Chinese traditional pipa ensemble. The composition was first found in "Hua Qiuping's Pipa Profiles", and the composer and the time of creation are no longer tested. Before learning and playing the song, First of all, we should have a rational understanding of the background knowledge and creative purpose of the music. The song is based on the historical background of the struggle between the late Qin Dynasty and the Chu and Han dynasties, Liu Bang and Xiang Yu Gaixia, and uses the unique performance techniques of the pipa. To describe Xiang Yu in all aspects of the embattled farewell scene with Yu ji. 
The music brings together the eternal themes of war, love, parting, death and so on, and takes the pain and the tragedy of death, which arouses great sympathy from the audience, as the main body, and takes the exciting sound produced by the various playing techniques of the pipa as the main body. With the tragic color of moving heart, it creates the perfection of music and makes it a treasure in the treasure-house of Chinese national music.

The song tries to create a kind of music atmosphere of "sad beauty". To create a hero image of the Western Chu hegemon, "Li Bu Shan Xi Xi Shi Shi Shi". This is obviously different from the "Ten facades ambush" written in the same historical theme [7]. Only the background knowledge of the music is known. With the comprehensive and rational cognition, we can grasp the emotional latitude of the music playing as a whole and lay the foundation for our rational performance.

Abstract thinking is helpful to analyze the musical structure of pipa music, the internal relationship between the phrase and paragraphs and paragraphs, and to memorize them completely, and finally to play the music correctly and rationally.

After numerous explorations and studies, previous scholars have formed the basic theory, basic playing skills and methods of pipa music. This knowledge is the theoretical basis of pipa playing, and their mastery mainly depends on abstraction and image thinking. Musical memory plays an important role here.

When a player with a certain theoretical knowledge of pipa music is faced with a new pipa music, he must, first of all, understand the relevant background knowledge of the music under the control of abstract thinking. He then mobilizes the music knowledge he already has in his brain to carefully read the music score, to analyze the composition of the music, to analyze the interrelationship between the passage and the passage, and between the passage and the paragraph. In order to form a rational impression on the music is as a whole and in part. After careful analysis, the performer will rely mainly on abstract thinking, and through musical memory, he will put the notes and rhythms in the music score. The internal connections between emoticons and musical structures are implanted into the brain.

In this way, the player can mobilize the memory of the brain, grasp the structure of the music and the internal relationship between the paragraphs reasonably, and logically express the emotion contained in the part and the whole of the music.

For example, "Dance of the Yi people" is a famous pipa dance composed by Mr. Wang Hui-ran on the fifth of 20th century and 60s. The music is compiled according to the folk tones of the Yi people in Yunnan, with a beautiful lyrical melody and a rugged strong rhythm. Depicts the enchanting night of the Yi village and the happy scenes of the young dance. The music is a trilogy structure with an introduction and an end.

It combines Chinese traditional music multi segment body gradually evolved, the development of technique, make the transition contrast between paragraphs appear natural, making music with strong ethnic style: Music in the introduction yudiao on the slow start growing fast; then, with the double chord melody in Pipa bass music, the first part the music; the second part of the music is Allegro, is the variation and development of the theme of the music; the third part of the music, the first part is introduction and reproduction of adagio music; finally, music is coming to an end.

Only by clarifying the structure of the composition and the internal connection between the three paragraphs, can the emotion contained in the part and the whole of the music be logically displayed under the action of music memory.

Abstract thinking is also helpful for the proper control of pipa playing.

Abstract thinking can also help to control the pipa playing moderately. The control mainly includes two aspects: one is the performance skills; the other is the performance emotion.

It is the comprehensive ability and cultural quality that the pipa performer must possess to grasp the style, characteristic, emotion color and expression form of the pipa music deeply, so how to grasp the above elements? Before we play a pipa piece, we must do a good job in the following aspects. One is to grasp the emotional color of the music. To find out that the inner emotions of each passage are depressed, sad, happy, rough, excited, enthusiastic, deeply analyze, study and understand the different musical images and emotional expression methods of each paragraph. 
In terms of performance skills, coordination with the performance of emotions is particularly important in performance, especially when we play a more intense piece of music, which usually leads to excessive muscle strain due to the push of natural emotions. The loss of the natural muscle state required by technical action affects the expression of sound and the true expression of emotion.

\section{Analysis Role of Abstract Thinking in Pipa Performance}

Strengthening students' artistic accomplishment is an important way to improve students' musical feeling and emotional experience [8]. Through the students' listening, watching, memory and other ways, it further enriches the students' artistic expression means and musical cells. To successfully play pipa works, we need to strengthen students' artistic accomplishment. On the one hand, we need to actively learn from the purification of various schools, schools, and various musical instruments. While inheriting and developing pipa folk music, we should actively absorb the strengths of other countries and make use of them. On the one hand, we need to strengthen the understanding and mastery of related art fields.

As far as performance emotion is concerned, it is easy to appear two wrong tendencies in actual performance: excessive exaggeration and ostentation or excessive restraint and inflexibility. The former belongs to the arbitrary expansion of self-expression desire and imposes the consciousness of individuality on the music works; the latter is a lack of creative, lifeless mechanical performance, just the original copy of the music or surface description.

In actual pipa teaching and pipa playing, it is not difficult to find that many students play very skillfully, accurately in tone and rhythm, but the audience will find it as dull as boiled water and unable to get along with the audience. There are many factors that lead to this result, but the most important thing is that the expression of emotion is not enough, and in the process of pipa performance, there is a lack of certain emotional input.

Both tendencies are contrary to the aesthetic principles of musical performance and the composer's willingness to create. Joseph Barowitz, an American pianist and critic, once said, "A successful performer must be the personality of the performer and the composer." The personality of a whole, It's not that either side is oppressed. There's always a delicate balance between the two personalities. Often the right balance appears when the art of playing is at its peak.

In order to be "just right," you need to have a deep understanding of the music itself and to grasp its emotional needs, as well as a variety of skills that are appropriate to the performance of the music, and that is the only way to do so [3]. Ability to control the moderate aspect of musical performance freely and easily. All these depend on abstract thinking [4].

For example, the pipa song "Weihe River situation" is a piece of music with a high demand for emotion, which shows the author's deep nostalgia for the homeland of the three Qin Dynasty. It has a strong local style of Guanzhong. The main melody is composed of the bitter tunes in the Qin Opera. The music is euphemistic and deep, graceful and lyric, which makes the listener feel nostalgic for his hometown very naturally.

Coupled with the left hand and push pull string Pipa vibrato skill, make melody more elegant, $\mathrm{P}$, affectionate and continuous meaning. Touch one deeply in the heart, playing the song, if only stick mark from score "push" and "pull", "Yin", "rubbing" playing skills, but no expression of emotion person, will appear dry, can not cause the listener's emotional resonance.

However, too much exaggeration to express one's inner feelings, to impose one's own consciousness on the work, and not to consider the composer's creative intention and the emotion to be expressed, will only give one a feeling of performing for the sake of showmanship. Therefore, according to abstract thinking, appropriate control of performance skills and emotional release is particularly necessary.

Pipa playing is a musical activity that integrates emotion and action skill. Therefore, in the performance, the performer should first combine his own emotion with the emotion expressed by the music, and understand the emotion expressed in the work as truthfully as possible. At the same time, we should distinguish between ourselves and the characters in the works, and distinguish the muscle state in the natural emotional state from the muscle state required by the technical action, so that we can 
maintain a normal playing state. The rational expression of technical action [5], it is which mainly depends on abstract thinking for rational control.

\section{Summary}

Therefore, music abstract thinking, with the help of strict logical relationship and pure rational mode of thinking, introduces pipa playing into a scientific, systematic and standardized track. Under the guidance of abstract thinking, only the performer can fully understand the relevant knowledge of the music, establish the general emotional and rational latitude of music playing, and lay a solid theoretical foundation for the proper display of image thinking and inspiration thinking.

\section{References}

[1] Zhang Xian-ling, Yang He Ji, Hong Kong: Wei Ke Hua Cui Publishing House Press, 2003:1-11.

[2] Zheng Xingsan. On the thinking Mode of Piano performance. Journal of the Central Conservatory of Music,1996, (2).

[3] Zheng Xingsan, selected works of Piano Music, Xiamen: Xiamen University Press, 2002: 42-61.

[4] Maazel,Shen Guozhang, A few theoretical questions. Journal of Foreign Music Materials, 2010, vol. 26, No. 19.

[5] Cheng Jianping, An introduction to Control in Music performance, Journal of Wuhan Conservatory of Music, 1999, (3).

[6] Lan Lan. On emotional expression and cultivation in pipa performance. Grand stage 2009, 08

[7] Li Xiaoming. Pipa performance skills and emotional performance. Chinese Music Education, 2012.9

[8] Xu Caiping. An Analysis of the Musical feeling and artistic expression in Pipa performance. Journal of three Gorges University (Humanities and Social Sciences Edition, 2011,05 